\title{
Forum CC: caisse unique
}

Le thème de la caisse unique a suscité de nombreuses lettres de lecteurs. Certaines d'entre elles concernent plus spécialement la liberté de vote décidée par la Chambre médicale, et le président de la FMH, Jacques de Haller, y répond ci-dessous.

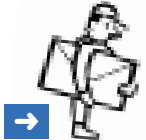

\section{Cavete Collegae}

Mit grossem Erstaunen, um nicht zu sagen Bestürzung, habe ich in der Berner Zeitung vom 20. Januar 2007 gelesen, dass bei einer Abstimmung zum jetzigen Zeitpunkt mehr als 50\% der Hausärzte einer Einheitskasse zustimmen würden. Ich bitte alle eindringlich, nochmals ihre Meinung zu überdenken. Glaubt irgendjemand von Euch wirklich, dass wenn Coop und Migros sich zu einem Staatsbetrieb zusammenschliessen würden, von Staatsangestellten verwaltet, sich für die Konsumentinnen und Konsumenten in unserem Land irgendetwas verbessern würde oder die Waren günstiger würden? Bereits heute spielen die grossen Kassen ihre Macht mehr als deutlich aus. Wenn sich nun alle Krankenkassen $\mathrm{zu}$ einem einzigen Staatsbetrieb zusammenschlössen, und dieser von jemandem wie Herrn Couchepin oder Herr Manser geleitet würde, kann sich irgendjemand von Euch vorstellen, dass es für uns Ärztinnen und Ärzte besser würde? Die Krankenkassen geben heute etwa 1\% ihres Budgets für Werbung aus. Dieses eine Prozent würde sicher wegfallen. Aber auch jedes Bemühen um Versicherte würde wegfallen und jede Anstrengung, sich gegenüber der Konkurrenz positiv abzuheben. Eine solche Megakasse hätte jede Macht, uns alle Bedingungen zu diktieren. Dass diese Macht auch benützt würde, ist nicht zu bezweifeln. Die Berechnungen darüber, dass diese Megakasse insgesamt auch wesentlich mehr kosten würde, und dies insbesondere den Mittelstand, liegen ebenfalls vor und können von jeder und jedem studiert werden (wurde uns allen Anfang Jahr zugestellt). Ich bitte Euch deshalb eindringlich, eine Einheitskasse abzulehnen!

Dr. med. A. W. Gerbig, Köniz

\section{Es lebe die Einheitskasse!}

Das Bundesgesetz über die Krankenversicherung (KVG) ist ein schlechtes Gesetz. Durch Volksabstimmung wurde es unglücklicherweise mit 51\% Stimmen dafür und $49 \%$ dagegen angenommen: das heisst mit nur 30000 Stimmen Unterschied.

\section{Bilanz des KVG}

Als Westschweizer Präsident des Komitees gegen das KVG hatte ich vor der Abstimmung die schlechten Seiten dieses Gesetzes aufgezeigt. Ich hatte meine Argumentation in der Hinsicht zusammengefasst, dass, wenn das Gesetz angenommen würde, die Leute mehr zahlen und weniger gut gepflegt würden.

Wir sind nun soweit, und an ein Zurück zu denken wäre illusorisch. Man muss also versuchen, die perversen Auswirkungen dieses schlechten Gesetzes so gut wie möglich abzuschwächen.

Ideal wäre, die Versicherungspflicht abzuschaffen, was zur Folge hätte, dass die Kassen in Wettbewerb träten, ihre Beiträge reduzieren müssten, sonst die Leute die Versicherung verlassen würden. Aber das würde als antisozial betrachtet.

\section{Demokratie in Gefahr}

Alle politischen Versuche, das Gesetz zum Vorteil der Versicherten zu ändern, sind auf die Ablehnung der Politiker, von denen ein grosser Teil in den Verwaltungsräten der Kassen sitzen oder sassen, gestossen.

Für diese Manipulation der Demokratie gibt es nur eine einzige Lösung: die Einheitskrankenkasse.

\section{Die Lösung: die Einheitskasse}

Diese Lösung würde erlauben, mit den Reserven der Kassen Schluss zu machen, würde die Werbekosten, die Löhne der Direktoren und anderer Leiter sowie die Vergütung der Verwaltungsräte vermeiden.

Sie würde die jährlich anfallenden Verwaltungskosten von santésuisse von 23 Millionen Franken einsparen sowie alle mit unseren Beiträgen bezahlten Instanzen, die dem Gesundheitswesen nichts anderes als Verwaltungsplackerei bringen. 
Das würde auch die Zahl der Angestellten der Krankenkassen begrenzen, die fast die Zahl der Ärzte übersteigt!

So würden die Beiträge deutlich vermindert, im Gegenteil zu dem, was die Gegner der Einheitskasse behaupten.

\section{Abwesenheit der Transparenz der Krankenkassen}

Betrachtet man die Erhöhung der Gesundheitskosten und vergleicht man sie mit der Erhöhung der Beiträge, so kann man nur beeindruckt sein von der Jahr zu Jahr grösser werdenden Diskrepanz. Die Gesundheitskosten steigen weniger als die Beiträge, was bedeutet, dass immer mehr Geld von den Kassen zu etwas anderem benützt wird, als die Kosten zu erstatten.

Die Beiträge sollten aber doch nur zur Erstattung der Kosten dienen.

\section{Einheitskasse $=$ Durchsichtigkeit}

Mit der Einheitskasse wird dies der Fall sein, die Verwaltungskosten würden auf ein Minimum reduziert und die Führung in Händen einer Kommission liegen, die aus Versicherten, Pflegern und Parlamentariern zusammengesetzt wäre.

Diese Kommission hätte demnach die Verantwortung, aufgrund medizinischer Kriterien über neue diagnostische und therapeutische Massnahmen zu entscheiden, und nicht, wie gegenwärtig, Angestellte ohne medizinische Kenntnisse nur nach finanziellen Kriterien, ohne das Interesse der Kranken in Betracht zu ziehen.

\section{Diktatur der Krankenkassen}

Die Behauptung, dass die Einheitskasse ein Monopol der Krankenversicherung schaffen würde, ist falsch, denn dieses Monopol existiert ja schon. Die Kassen haben einen Dachverband «santésuisse», der Entscheidungen trifft, die dann die Kassen ausführen.

Und was machen die Versicherer gegen die Einheitskasse? Sie haben ein Vermögen von mehreren Millionen angehäuft, das sie von den Beiträgen der Basisversicherung abgeschöpft haben. Ein Volkswirt wurde engagiert, um eine Studie zu veröffentlichen, in der wortwörtlich steht, was die santésuisse seit mehr als 10 Jahren ständig wiederholt: Gebt uns die Macht durch Abschaffung der Vertragspflicht. Sie haben ein Komitee «Gesundheit für alle» gegen die Einheitskasse geschaffen, in dem - welch ein $\mathrm{Zu}$ fall - der Präsident von santésuisse und andere Mitglieder der Verwaltungsräte der Kassen sitzen und das von santésuisse finanziert wird.

Die einzige Möglichkeit, dieses perverse System zu ändern, ist die Einheitskasse.
Die Kassen müssen aber gezwungen werden, dem Volk alle seit Jahren gebildeten Reserven gänzlich zurückzuerstatten, dazu gehören auch die Anlagen, die als «amortisiert» gelten. Das würde erlauben, die Beiträge nicht nur zu verringern, sondern sie wahrscheinlich auch mehrere Jahre lang abzuschaffen.

\section{Finanzierung}

Was die Finanzierung der Einheitskasse betrifft, sieht die Initiative vor, die Beiträge nach den wirtschaftlichen Möglichkeiten der Versicherten festzulegen. Wie für alle Verfassungsinitiativen handelt es sich um einen Grundsatz, und das Parlament wird ihn durch ein Gesetz präzisieren. Man kann verschiedene Modelle ersinnen. Aber im Gegensatz zu heute wäre es der Bevölkerung immer möglich, gegen ein anzuwendendes Modell ein Referendum zu beantragen, wenn ihnen dieses nicht gefallen sollte.

\section{Zum Schluss}

Es ist klar, dass man nach Annahme der Initiative gegen den Staat kämpfen muss, aber es ist besser, demokratisch gegen den Staat zu kämpfen, als noch lange die Diktatur der Krankenkassen zu erleiden.

Die Folge dieser Diktatur ist für uns der ganze Verlust unserer Freiheit und für unsere Patienten Beitragserhöhung und immer weniger gute Pflege.

Die Einheitskasse ist besser als der heutige Zustand.

Dr. med. Rudolf Mayer, Pully

\section{Einheitskasse beim obligatorischen KVG}

Da wird mit populistischer Argumentation von Steuern anstatt von Prämien gesprochen und mit Zweiklassenmedizin und Rationierung gedroht: Nichts ist davon im Initiativtext enthalten und kann von diesem auch nicht abgeleitet werden! Hingegen werden soziale Prämien, abgestuft nach wirtschaftlicher Leistungsfähigkeit, verlangt. Die Prämienhöhe ist in der Initiative nicht festgelegt und muss erst noch ausgehandelt werden. Ein Steuersystem wurde bereits früher abgelehnt und steht nicht zur Diskussion. Das obligatorische Krankenversicherungsgesetz wird unabhängig von Annahme oder Ablehnung der Initiative durch das Parlament bestimmt und ist referendumsfähig.

Mit nur einer Kasse werden gute und schlechte Risiken gleicherweise adäquat berücksichtigt, so dass die heutigen unfairen Abwerbepraktiken aufhören - unter erheblichen Kosteneinsparungen. Gemäss Initiative wird die Kasse von einem Verwaltungsrat geführt und von einem Auf- 
sichtsrat kontrolliert. In beiden Institutionen sind die Leistungserbringer, die Patientenvertreter und die Behörden paritätisch zu genau gleichen Teilen vertreten. Können wir je mit den jetzigen Kassen auf diesen Vorteil hoffen?

Wenn wir uns wirklich für das Wohl der Patientinnen und Patienten einsetzen und dem Prinzip «Gesundheit für alle» nachleben wollen, dürfen wir nicht nur im Gesundheitsmarkt für unsere existentiellen Bedürfnisse kämpfen, sondern müssen uns ehrlicherweise auch solidarisch an leistungsgerechten Prämien beteiligen und für diese einstehen. Dies wirkt einer Zweiklassenmedizin entgegen, die wir zwar ohnehin schon haben, aber in unserem freiheitlichen System begründet ist. Mit leistungsgerechten Prämien muss auch nicht befürchtet werden, defizitär zu werden, weil vom notwendigen Prämienvolumen ausgegangen wird und erst danach die Aufschlüsselung der Einzelprämien erfolgt. Schon gar nicht kann irgendein System dem Thema Rationierung ausweichen. Schliesslich können wir nicht über Jahrzehnte dank unaufhaltsamer Forschung und medizinischer Fortschritte andauernd mehr Leistungen erbringen und noch glauben, dass dies nicht an eine unbezahlbare Grenze stossen wird, oder dass diese mit einem guten Krankenkassensystem vermieden werden kann!

Dr. med. Georg Hinz, Mönchaltorf

\section{Votation du 11 Mars 2007: concurrence ou solidarité?}

Les opposants à la caisse unique ne vont pas lâcher facilement leur os: ces caisses multiples peuvent être de fantastiques vaches à lait financières. De leur vocation initiale de caisses mutuelles, elles sont passées à l'état de sociétés anonymes, devant obéir bien sûr aux lois (la LAMal en particulier) mais avant tout aux lois de l'économie privée: il faut dégager du bénéfice et de la rentabilité. Ce qui est valable pour les assurances privées tend à le devenir pour les assurances sociales. Cette dérive progressive est gravissime quand on voit déjà maintenant des soins de bases refusés quand le paiement des primes a du retard. Nous voilà 50 ans en arrière quand il fallait déposer Fr. 300.- à 500.- avant de passer les portes de l'hôpital. Beau progrès! Les radicaux du début du $20^{\mathrm{e}}$ siècle furent les initiateurs des lois sociales afin de niveler les inégalités criantes héritées du passé. Ceux d'aujourd'hui, notamment Pascal Couchepin, devraient relire leurs déclarations avant de crier concurrence, libéralisme, économie de marché. Le vote du 11 mars marquera le choix de la Suisse entre le lobby de l'économie privée et la cohésion nationale. De «Un pour tous, tous pour un», va-t-on passer à «Chacun pour soi, tous pour l'actionnaire»? Solidarité ou égoïsme forcené?

Il est clair que notre système de santé actuel a ses tares, mais il est aussi un des meilleurs du monde. A nous de lui laisser ses chances de conserver son rang. Entre les patients qui abusent des prestations et les médecins, hôpitaux, prestataires divers, qui surfacturent, les possibilités d'économies sont nombreuses. Il faut que chacun se responsabilise face au problème. Le Danemark est à cet égard un modèle en Europe. L'évolution technique de la médecine est aussi un facteur important des coûts: chacun souhaite profiter de la technologie la plus avancée et chaque prestataire de cette technologie souhaite la rentabiliser au mieux. Rappelons-nous les premiers scanners. Le personnel des soins a beaucoup changé: depuis les bonnes sœurs à cornettes vivant d'un quignon de pain, de soupe et de prières, il a fallu s'adapter aux technologies sans cesse mouvantes et nécessitantes des formations de haut niveau. Le réseau de santé Suisse est à revoir dans son ensemble, les doublons sont nombreux et les sensibilités locales particulièrement chatouilleuses. Ce serait une des premières tâches de la caisse unique: Etablir un réseau de santé cohérent, économique et solide. Les caisses actuelles n'ont pas ce pouvoir si ce n'est de dire qu'une appendicite coûte là 2500.- et ailleurs 3500.- Donc toutes les appendicites se feront là où elles coûtent le moins cher. Cette vision uniquement économique des faits n'est pas crédible. L'âge des patients, leur état général, leur mode de vie (meilleur à la campagne qu'en ville, faut-il le rappeler?), etc.

Le vote du 11 mars 2007 est un vote crucial: celui d'un choix de société: sociale ou économique. Quel avenir voulons-nous pour nos enfants, celui d'une société égoïste et soumise aux lois de l'économie privée, ou celui d'une société solidaire et responsable, offrant une garantie de soins à tous? Et si votre vote est guidé par votre porte-monnaie, sachez que personnellement, je paierai environ 400.- de plus par an avec fierté et responsabilité pour laisser une société digne et sociale à nos enfants comme le voulaient les radicaux du début du $20^{\mathrm{e}}$ siècle et comme nos parents nous l'ont laissée. Ne galvaudez pas des acquis péniblement obtenus. Et, en cas de doute, vous pouvez sans crainte glisser votre oui dans l'urne, les critiques de l'économie face à la caisse unique sont essentiellement la crainte de laisser passer le «juteux» marché de la santé dans le domaine public.

Nom connu de la rédaction 
Stellungnahme der FMH zur Einheitskasse Das Argumentarium der FMH zur Einheitskasse hat mich enttäuscht [1]. Die FMH ist in einer eminent wichtigen Frage zu einer eindeutigen Stellungnahme auch nach langem Palaver nicht fähig. Es werden Argumente aufgeführt, die gar nicht zur Initiative gehören. Der Widersinn einer staatlichen vom gleichen Staat kontrollierten Kasse wird dargelegt. Man wagt aber nicht, so weit zu denken, dass die Einheitskasse noch viel mehr Macht über die Ärzte haben würde als die jetzt bestehenden Versicherungen. Das Axiom, dass die gleichzeitige Betätigung im Grundversicherungsbereich und im Zusatzversicherungsbereich schädlich sei, wird nicht belegt. Man kommt doch dazu, zu erkennen, dass die Einheitskasse ein Monstrum wäre. Schliesslich verlangt man noch mehr Steuergelder, was mit der Vorlage nichts zu tun hat.

Im farbig unterlegten Kasten hält zwar die Ärztekammer kurz und bündig fest, dass die Vorlage keine Probleme löse, sie ist aber nicht imstand, den logischen Schluss zu ziehen, nämlich sie abzulehnen. Ich bin überzeugt, die Mitglieder der Ärztekammer gehen in der Tätigkeit mit ihren Patienten nicht in gleicher Weise vor: Bei Verdacht auf Herzinfarkt lang über die Gallenblase nachdenken, den Urin untersuchen, Reflexe prüfen, über das Herz diskutieren und dann nichts machen, sondern zuschauen, was die Ambulanzfahrer tun.

Bis ich vor gut drei Jahren meine Zuweisungspraxis aus gesundheitlichen Gründen aufgeben musste, arbeitete ich mit vielen Kollegen zusammen. Sie alle waren freie Unternehmer und legten Wert auf diesen Status. Jetzt werde ich von ausgezeichneten Kollegen betreut, die glücklicherweise zur Selbständigkeit fähig sind. Bezüglich der FMH zweifelte ich zum ersten Mal, als ich in der NZZ das Gespräch zwischen Bundesrat Couchepin und dem Präsidenten der FMH las. Dr. de Haller postulierte darin, dass man endlich über einkommensabhängige Krankenkassenprämien sprechen sollte. Bundesrat Couchepin antwortete, dass die Ärzte keine Unternehmer seien. Bezüglich des Zentralpräsidenten hat er recht.

Es ist nicht überraschend, dass es in der Ärzteschaft unterschiedliche Ansichten gibt. In der gleichen Nr. 1 der SÄZ ist ein Leserbrief publiziert [2], in der 60\% der Waadtländer Ärzte für die Einheitskasse sind, mit umwerfender Logik sind dann 76\% dafür, dass die kantonale Organisation sich im Abstimmungskampf engagiere. Die Meinung der 16\%, die zwar nicht für die Einheitskasse sind, aber für das Engagement im
Abstimmungskampf sind, würde mich interessieren. Da haben wir immerhin eine Stellungnahme. Folgt die gesamte Ärzteschaft der Schweiz den Waadtländern, dann ist sie halt am Schluss für die Verstaatlichung der Medizin, aber wenigstens nicht unklar wie die Ärztekammer.

Das Argumentarium dürfte dem Ansehen der Ärzteschaft nicht nützen.

\section{Dr. med. Peter Corrodi, Küsnacht/ZH}

1 FMH. Initiative für eine Einheitskasse. Stellungnahme der Ärztekammer vom 14./15. Dezember 2006. Schweiz Ärztezeitung. 2007;88(1):7-9.

2 Borzykowski M. Caisse-maladie unique? Bull Méd Suisses. 2007;88(1):15.

\section{Stellungnahme der Ärztekammer zur Initiative für eine Einheitskasse}

Die Ärztekammer hält fest, dass mit der Initiative die Probleme des Gesundheitssystems nicht befriedigend gelöst werden können und dass auf die Erwartungen der Ärzteschaft keine angemessenen Antworten gegeben werden [1]. Die logische Konsequenz wäre somit die Ablehnung der Initiative. Eine Stimmfreigabe wird in der Öffentlichkeit nicht verstanden. Wenn wir uns zu einem Thema, welches jeden einzelnen von uns bei der täglichen Arbeit betrifft, nicht äussern, sind wir weder für Politiker noch für Vertreter von Krankenkassen und Versicherungen noch für unsere Patienten ernstzunehmende Gesprächspartner. Bei einer so wichtigen Frage muss die Ärzteschaft auf die eine oder andere Art Stellung nehmen. Stimmfreigabe mit der Botschaft, es ist uns egal, was mit uns passiert, ist die schlechteste aller Lösungen. Die Ärztekammer als Gremium macht sich lächerlich, und wir Ärzte werden nicht mehr ernstgenommen.

PD Dr. med. M. G. Schwöbel, Luzern

1 FMH. Initiative für eine Einheitskasse. Stellungnahme der Ärztekammer vom 14./15. Dezember 2006. Schweiz Ärztezeitung. 2007;88(1):7-9.

\section{Prise de position de la chambre médicale sur la caisse unique}

Dans sa prise de position à propos de la caisse unique, la Chambre médicale craint l'apparition d'un «monstre administrativo-financier» [1]. Ces craintes sont tout à fait disproportionnées, notamment en comparaison aux bénéfices escomptables. Les différentes caisses sociales déjà existantes (comme l'AVS et la SUVA) fonctionnent bien et ne sont pas les monstres que l'on 
nous décrit. Les comparaisons avec les pays voisins, comme la France, sont inappropriées car tout le fonctionnement de l'administration y est différent et les sommes dépensées pour la santé y sont plus faibles qu'en Suisse.

On oublie par contre les bénéfices attendus:

- Pour l'ensemble de la population: suppression des frais de publicité, des frais liés aux changements de caisse, de la structure de contrôle des différentes caisses, de la structure de compensation des risques ...

- Pour les médecins: pas besoin de redemander régulièrement le nom de leur caisse maladie aux patients, arrêt de la multiplication de négociations avec différentes caisses proposant chacune leur système, simplification des procédures, transparence des chiffres ...

\section{Réponse}

Je crois qu'il est important de bien considérer la prise de position de la Chambre médicale du 15 décembre dans son intégralité, et de bien situer son contexte.

C'est une prise de position intelligente, nuancée, et riche de contenu - vous la retrouverez sur notre site internet à l'adresse www.fmh.ch communiqués de presse/prises de positions/ informations et dans l'édition no 1/2007 du Bulletin des médecins suisses.

Elle prend en compte la diversité de la FMH, des opinions qu'on y rencontre, et de la culture politique qui y règne, mais ne s'arrête pas à ces difficultés et propose une réflexion et des projets solides et consistants.

Autrement dit, dire simplement «oui» ou «non» à cette initiative n'aurait pas rendu compte de la complexité du sujet, qui concerne certes la caisse unique en tant qu'institution mais aussi par exemple la séparation de l'assurance sociale des assurances complémentaires privées, ou la démocratisation des structures de direction des caisses.

Et dire simplement «oui» ou «non» n'aurait pas non plus permis à tous les membres ou à
Contrairement à l'avis de la chambre médicale, les différences de sensibilités culturelles pourront être mieux prises en compte grâce à la caisse unique. Le système prévu imagine des offices locaux sur le modèle de l'AVS. Ils permettraient un contact de proximité. Ceci irait à l'inverse de la tendance actuelle où les décisions sont de plus en plus prises par les directions centrales des caisses sans grande connaissance des spécificités locales.

Enfin, la caisse unique garantira la liberté de contracter, que les caisses se dépêcheront d'introduire en cas de refus de l'initiative.

\section{Dr Michel Perrenoud, La Chaux-de-Fonds}

1 FMH. Initiative pour une caisse-maladie unique. Prise de position de la Chambre médicale des 14-15 décembre 2006. Bull Méd Suisses. 2007; 88(1):7-9.

toutes les sociétés de la FMH de se sentir pris en compte, eux/elles dont les positions sont si diverses.

Vous verrez aussi en relisant notre argumentaire que la liberté de vote décidée par la Chambre médicale s'accompagne de revendications pressantes, qui feront l'objet d'une pression politique et médiatique importante.

Ces revendications visent à lutter contre les inacceptables conflits d'intérêts qui bloquent actuellement l'évolution du système de santé en liant l'assurance sociale obligatoire aux intérêts financiers des assureurs privés; et aussi à rendre plus démocratique l'administration d'institutions qui gèrent des dizaines de milliards de francs dans des comités simplement cooptés; ou encore à soutenir le développement des réseaux de soin, ou à favoriser l'esprit de partenariat entre acteurs du système.

Ainsi, la Chambre médicale a pris une position constructive et respectueuse de notre diversité - une position nuancée et intelligente!

Dr Jacques de Haller, président de la FMH 